\title{
The Hand-bot, a Robot Design for Simultaneous Climbing and Manipulation
}

\author{
Michael Bonani, Stéphane Magnenat, Philippe Rétornaz, and Francesco \\ Mondada \\ Michael Bonani \\ EPFL-LSRO, Station 9, CH-1015 Lausanne \\ michael.bonani@epfl.ch \\ http://mobots.epfl.ch/
}

\begin{abstract}
We present a novel approach to mobile object manipulation for service in indoor environments. Current research in service robotics focus on single robots able to move, manipulate objects, and transport them to various locations. Our approach differs by taking a collective robotics perspective: different types of small robots perform different tasks and exploit complementarity by collaborating together. We propose a robot design to solve one of these tasks: climbing vertical structures and manipulating objects. Our robot embeds two manipulators that can grasp both objects or structures. To help climbing, it uses a rope to compensate for the gravity force. This allows it to free one of its manipulators to interact with an object while the other grasps a part of a structure for stabilization. Our robot can launch and retrieve the rope autonomously, allowing multiple ascents. We show the design and the implementation of our robot and demonstrate the successful autonomous retrieval of a book from a shelf.
\end{abstract}

\section{Introduction}

Service robotics has a large economic potential [1]. In its applications, if robustness and flexibility are dominant requirements, collective robotics is a promising paradigm $[2,3]$. Because collective robotics distributes the work to multiple robots, it requires different control, more hardware units, and usually more engineering resources than the approaches based on single robots [4]. Yet the physical redundancy between the robots enables a high availability. Moreover, their modularity provides adaptation to changing needs and situations. These unique features might outstrip the drawbacks of collective robotics eventually.

To implement service robotics tasks successfully, groups of robots must be able to manipulate objects in the environment. These objects can be located in altitude, and robots part of a collective are small. Therefore, these robots must be able to climb the environment to fetch the objects. This paper proposes a design concept and demonstrates a working implementation of a climbing robot that performs manipulation tasks. 
In a heterogeneous group, as proposed by the Swarmanoid project [5], our robot is only responsible for manipulation of objects in altitude. While on the floor, it relies on other types of robots for mobility. These robots would have different hardware, in particular, they would be able to self-assemble with our robot and move it on the ground [6]. Such task and hardware specialization allows the best use of the capabilities of each type of robots while self-assembling provides the synergy to compensate for the limitations of each individual type.

\section{State of the art}

The ascension of vertical surfaces is a difficult problem: it requires a lightweight robot with strong actuators, which results in a sensitive mechatronic design. For this reason, few systems combine the abilities of climbing and manipulation. Researchers in the field of space manipulators - where robots move in microgravity - have already studied this combination [7].

Some robots require an adaptation of the environment, such as in [8] where the robot needs custom-tailored docks to attach. To remove this limitation, several works have explored vacuum adhesion. For instance, [9] proposes a mediumsize robot (diameter of $50 \mathrm{~cm}$ ) for inspection of large surfaces. A vacuum system provides the adhesion force while three wheels allow the robot to move on the surface. To scan the surroundings of the robot, an inspection sensor is attached to a parallel arm. This system does not allow manipulation of external objects.

The Alicia ${ }^{3}[10]$ is a larger robot (length of $1.3 \mathrm{~m}$, weight of $20 \mathrm{~kg}$ ) which also uses pressure for adhesion. It consists of three attachment units linked by an articulated arm. Each unit contains its own vacuum generator and displacement wheels. Although the design of this robots is focused on climbing, its arm-based structure could support manipulation capabilities.

Several authors have proposed large platforms for outdoor operations on buildings or industrial superstructures [11], often for specific applications [12]. These platforms could support the addition of manipulators; however their size makes them unsuitable for the type of application we consider.

There exist several robots that utilize some form of grasping to climb [1315]. Their grippers are custom-tailored to specific structures. Yet none of these robots can use their grippers to perform object manipulation.

To the best of our knowledge there is no small autonomous robot (few decimeters cube) that implements a combination of climbing and object manipulation.

\section{Design and implementation}

We propose a novel approach to mobile object manipulation for service in indoor environments. It takes advantage of synergies between climbing and object manipulation. This translates as the ability to climb common vertical offices structures such as shelves. Within these structures, the robot gets small objects such as lightweight books or compact discs. It then retrieves these objects to 


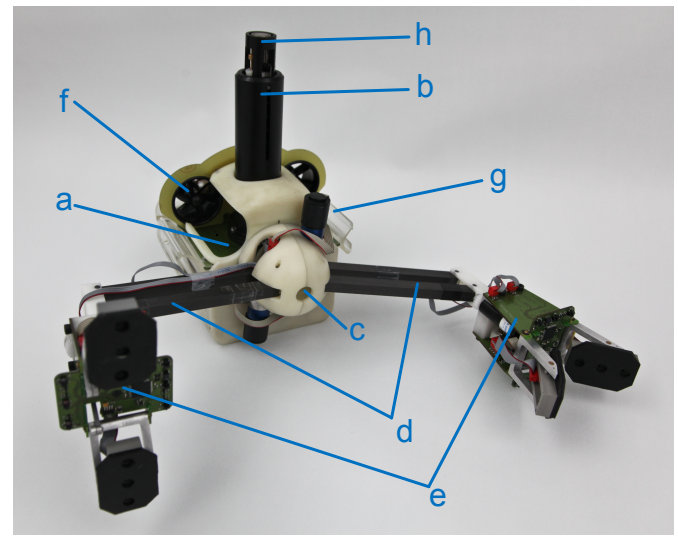

Fig. 1: Overview of the handbot. a: The body, containing in particular the battery, the rope launcher, and the head rotation motor. b: The tube of the rope launcher. c: The head, containing the two arms bending motors. d: The arms. e: The grippers, each containing two motors for rotation and grasping. f: Two fan to control the yaw when handbot hangs at the rope. g: Connection ring. h: Switchable magnet.

the ground and brings them to a specific location. We have implemented this approach in the hand-bot (Fig. 1) robot that we present in this article.

The biggest constraint for climbing under gravity is to provide the vertical lift force; we thus implement climbing by combining two techniques. Rolling a rope provides the vertical lift force while manipulators provide horizontal operations. The hand-bot fixes the rope to a ceiling and coils it around a reel. This mechanism is simple and can lift a large mass by using a strong motor; as shown by previous work [16]. To be autonomous, the robot must be able to attach its rope to a specific location, use it to climb, and retrieve it afterwards. We propose a launching mechanism based on a strong spring (Fig. 1b) that projects a magnet to the ceiling. Albeit this approach works only in environments with a ferromagnetic ceiling, it is well understood and reliable. Moreover, depending on the type of ceiling, other attachment mechanisms such as plungers are applicable. To be able to detach from the ceiling, the attachment must be switchable. We implement this feature using a magnetic switch that the robot can trigger with a specific infrared transmission.

When attached to the ceiling using the rope, the hand-bot has vertical mobility but is horizontally unstable. To stabilize and position itself on the horizontal plane, it needs manipulators to grasp the structure around it. The hand-bot has two arms, each with a gripper as manipulator (Fig. 1d,e). When using the two arms to climb, it maintains its stability all the time. It can also manipulate an object with one gripper while keeping the other one attached to the structure. That way, the hand-bot can manipulate objects precisely. Once on the ground, other types of robots can assemble with the hand-bot and displace it and the object it carries to a specific location. The other robots [5] attach to the hand-bot by grasping its translucent ring, which also contains 12 RGB LEDs.

\subsection{Rope launcher}

As we explained in the preceding sections, the rope provides the main lifting force of the hand-bot. Fig. 2A shows a photo of the mechanism of the rope 


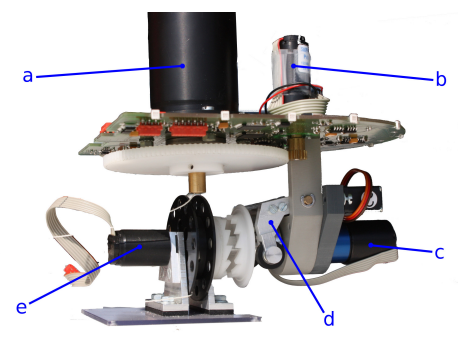

(A) rope launcher

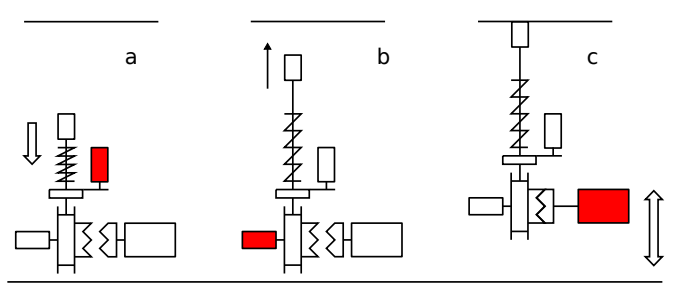

(B) rope launching sequence

Fig. 2: (A) Mechanism of the rope launcher. a: The launching tube containing a spring. b: The motor to compress the spring. c: The lifting motor. d: The servomotor clutch to engage the lifting motor. e: The motor directly connected to the reel, to coil the rope on magnet retrieval and to provide tension control. (B) Schematics of the launching sequence, filled rectangles show the active motor. a: A motor compresses the spring. b: A fast motor maintains tension in the rope while the spring launches the magnet. c: A clutch is engaged to let the strong motor lift the robot.

launcher and Fig. 2B shows the schematics of the launching sequence. To launch the rope, a motor compresses a spring using a small wagon. This wagon moves inside the launch tube and is driven by a worm gear. When the spring is fully compressed (at that point, it applies a force of $110 \mathrm{~N}$ ), the wagon hits the bottom of the tube and liberates the spring, which launches the rope, the magnet, and the detachment mechanism up to an altitude of 270 centimeters. Two different motors drive the rope: a strong one provides the main lifting force and a fast one controls the tension of the rope, during launch and retrieval. To switch between these two motors, a servomotor activates a clutch. During launch, the fast motor brakes the reel: this is necessary to prevent the formation of knots in the rope. The launching control program monitors the length of the uncoiled rope in real time. As soon as the magnet reaches the target altitude, the fast motor firmly coils back, which ensures the tension in the rope. If the magnet fails to attach, this action will coil back most of the rope and the hand-bot will know the result of the launch. If the launch succeeds, the servomotor engages the clutch so that the strong motor drives the rope, and provides the main lifting force for the hand-bot. This force is strong enough to lift the robot by itself.

If the hand-bot hangs freely at the rope, because it holds an object or has failed to grasp an element of structure, it can stabilize and orientate itself using an inertial measurement unit and two fans. The fans are located at the top of the body on both side of the rope launcher tube.

\subsection{Manipulators}

The hand-bot has two arms on its front side. They can bend/extend forward - independently - and rotate with respect to the robot body (Fig. 3). At the 


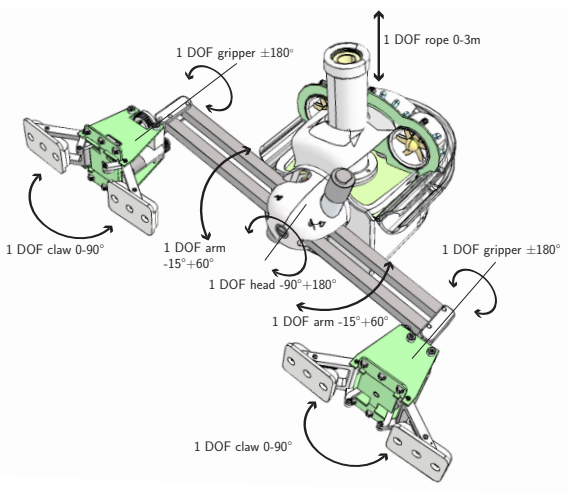

(A) kinematic of the hand-bot.

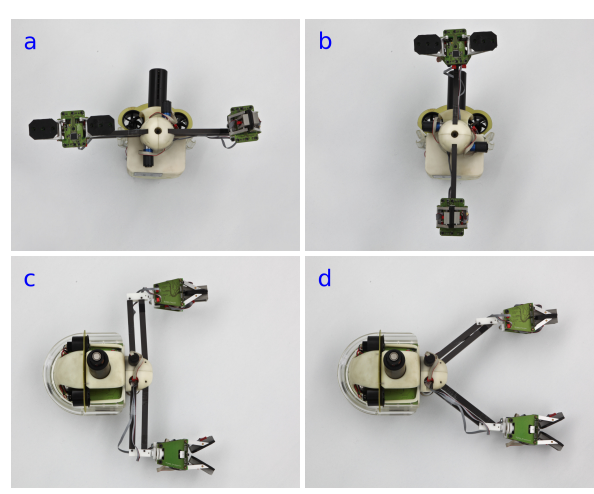

(в) extreme head and arms positions

Fig. 3: The degrees of freedom of the hand-bot.

end of each arm, the hand-bot has a gripper (Fig. 4A). Each gripper can rotate with respect to its arm and can open and close its claws. To grasp objects and structures of different thicknesses, the gripper claws have a parallel compliance mechanism. When no object is present, a spring maintains a large opening angle between the claws. Once the claws squeeze an object, the points of contact are different than the points of rotation which generates a moment that aligns the claws in parallel with the object. This provides a strong force over a large range of thicknesses. To control grasping, each gripper can detect structures and objects at close range using 12 infrared proximity sensors on its perimeter (Fig. 4B). These sensors have a range of 12 centimeters. In addition, the gripper has a VGA camera in its centner. This camera is capable of applying in hardware a Sobel filter to the image, which eases line and object detection.

\subsection{Electronics and control}

The motors of the hand-bot embed a position encoder, which provides control in position and speed. In addition, the driving electronics measure the currents in the motors, which provides control in torque. The electronics is built around modules containing a 16-bit microcontrollers (Fig. 5). Each microcontroller manages the sensors and actuators it is locally connected to. The modules communicate together using asynchronous messages. They exchange them over a CAN bus [17] using the ASEBA architecture [18, 19]. The ASEBA architecture distributes the processing locally by embedding a lightweight virtual machine inside each microcontroller. An integrated development environment compiles bytecode for these virtual machines out of a user-friendly scripting language. The development environment runs on a remote computer and provides the concurrent development - including debugging - of the programs on all the microcontrollers. Once complete, the program can be flashed into the microcontrollers for autonomous operations. 


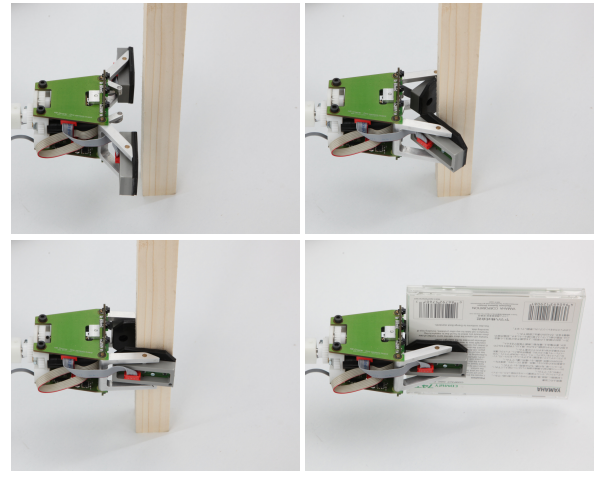

(A) passive compliance when grasping

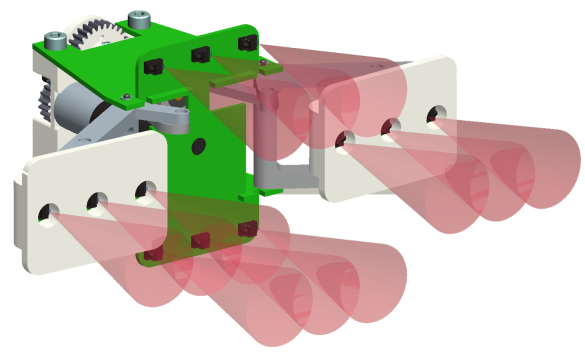

(B) locations of the infrared sensors

Fig. 4: The gripper can open at $90^{\circ}$ and close completely. When closing, its parallel compliance mechanism allows it to grasp objects of different thicknesses. The claws can apply a force up to $4 \mathrm{~kg}$ thanks to the high reduction of its worm drive.
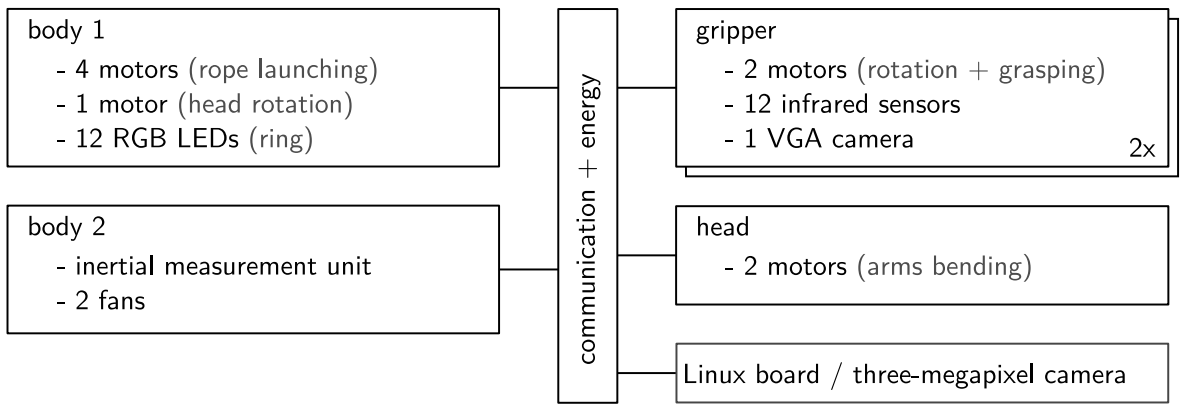

Fig. 5: Distribution of functions between the different microcontrollers. Each box represents a different microcontroller. The microcontrollers communicate together using asynchronous messages that they exchange over a CAN bus using the ASEBA architecture 
While the ASEBA architecture is powerful enough to implement any low-level autonomous behavior such as climbing a shelf; high level cognitive tasks such as locating a specific book using vision require more computational power. To fulfill this need, we will equip the hand-bot with an embedded computer running Linux, built around an ARM 11 processor and $128 \mathrm{MB}$ of RAM. This computer will also provides a Wifi connection and a three-megapixel camera located in the centner of the head. The experiments that we discuss in the next section run solely using ASEBA [18]; but the scenarios presented in the introduction will require this embedded computer.

\subsection{Precision of altitude control}

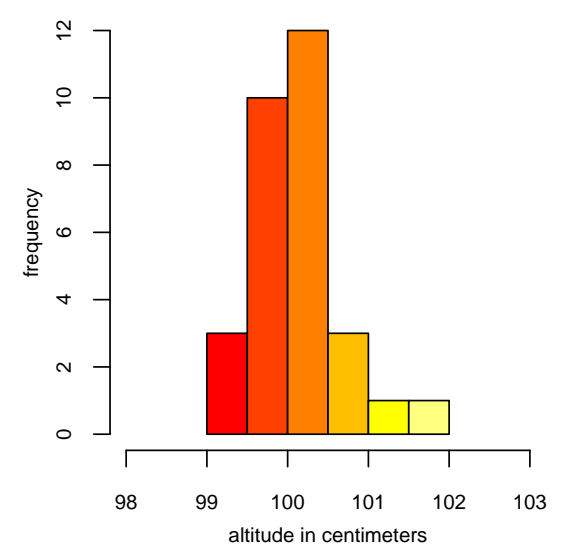

Fig. 6: Distribution of altitudes reached by the hand-bot for a target of $100 \mathrm{~cm}$ over 30 runs. The empirical mean is $100.2 \mathrm{~cm}$ and empirical standard deviation is $0.6 \mathrm{~cm}$.

The length of the coiled rope is the only information of the altitude of the robot. The experiment that we present in Sec. 4 considers the retrieval of a book located at a predefined altitude; which means that the precision of the altitude control is a determinant factor for its success. The hand-bot counts the rotations of the reel to estimate the length of the coiled rope. However, the rope might coil itself in different ways which might affect the effective radius of the reel. Moreover, the rope is elastic and its coiled length per reel rotation might change from launches to launches.

For these reasons, we characterize the error on the altitude. We let the handbot launch its rope and then elevate by coiling the rope until it reaches a target setpoint of 100 centimeters above the ground. We have repeated this sequence 35 times. In five of these trials, the launch was unsuccessful as the magnet did not attach to the ceiling. In the other 30 trials, the launch was successful. This results in a 86 percent success rate. Fig. 6 shows the altitudes that the handbot reached in these 30 trials. The mean altitude is 100.2 centimeters and the standard deviation is 0.6 centimeter; the biggest error is 2.0 centimeters. The 
altitude control using the rope is thus precise enough to climb to a target altitude and retrieve an object.

\subsection{Workspace}
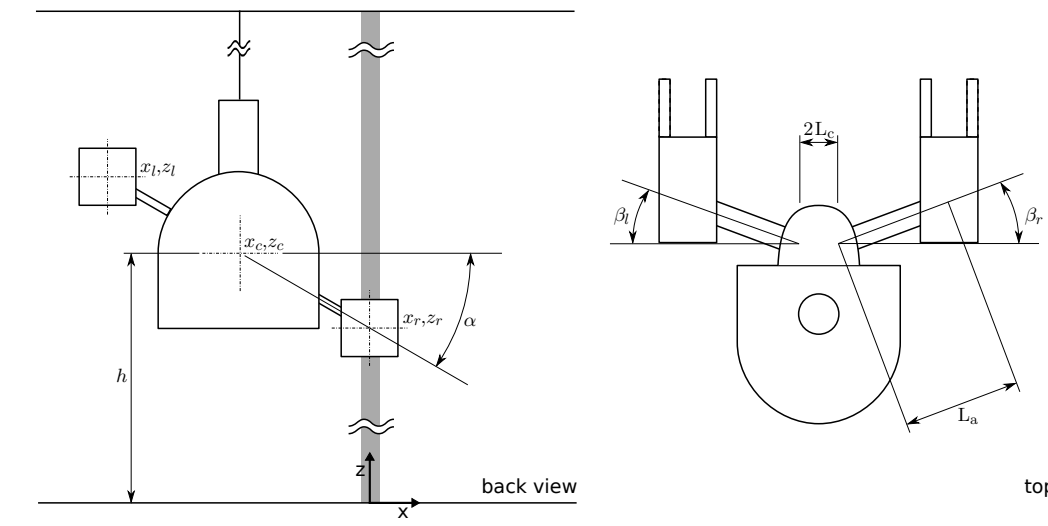

$\begin{array}{llll}l_{l}=\mathrm{L}_{\mathrm{c}}+\mathrm{L}_{\mathrm{a}} \cos \left(\beta_{l}\right) & l_{r}=\mathrm{L}_{\mathrm{c}}+\mathrm{L}_{\mathrm{a}} \cos \left(\beta_{r}\right) & x_{r}=0 & z_{r}=h-l_{r} \sin (\alpha) \\ x_{l}=x_{r}-\left(l_{l}+l_{r}\right) \cos (\alpha) & z_{l}=z_{r}-\left(l_{l}+l_{r}\right) \sin (\alpha) & x_{c}=l_{r} \cos (\alpha) & z_{c}=h\end{array}$

Fig. 7: Equations of the positions of the grippers given the length of the coiled rope, the head rotation, and the arms extensions. These equations apply when the right gripper is attached; they are symmetric when the left one is attached. The constants are the followings: $\mathrm{L}_{\mathrm{a}}=145 \mathrm{~mm}$ is the length of an arm. $\mathrm{L}_{\mathrm{c}}=$ $20 \mathrm{~mm}$ is half the length between the arms attachments points in the head. The variables measured by the robots sensors are: $\beta_{l}, \beta_{r}$ are the bending of the left, right arm. $\alpha$ is the rotation of the head. $h$ is the altitude of the centner of the head. The variables we look for are $x_{c}, z_{c}, x_{l}, z_{l}$, and $x_{r}, z_{r}$ : the positions of the center of the head, the left gripper, and the right gripper

The hand-bot climbs vertical structures by rotating its arms with one gripper fixed, and by switching grippers after each half turns (Fig. 8A). At the same time, the hand-bot coils the rope which provides the main lifting force. As the handbot only needs one gripper attached to provide stability, it can use the other one to manipulate objects. The hand-bot can compute the position of a gripper knowing the length of the coiled rope, the position of the head, and the extension of the arms (Fig. 7). While keeping a gripper attached, the hand-bot can reach objects located in a vertical disk (inner radius $18.2 \mathrm{~cm}$, outer radius $32.0 \mathrm{~cm}$ ) centered around this gripper. To access objects out of this disk, it must climb up or down, change the extensions of its arms, and then climb back to a position where it can access the object. In this way, the hand-bot can reach any objects located at maximum at 32.0 centimeters away from climbing pole (Fig. 8A). 


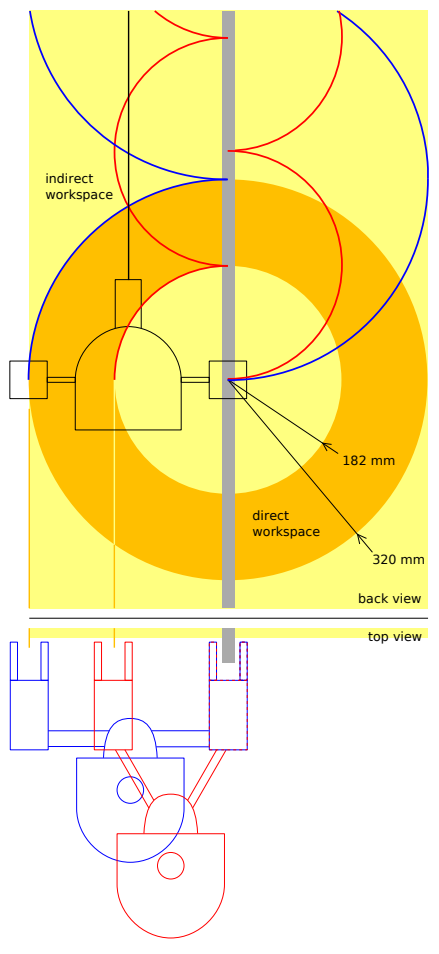

(A) workspace

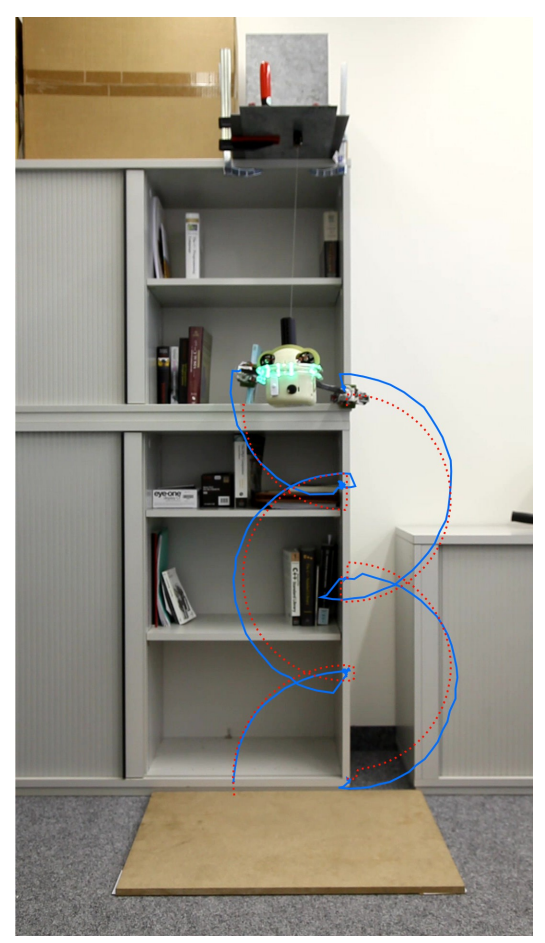

(в) climbing traces

Fig. 8: (A) Workspace of the hand-bot. The trajectories of the grippers for the two extreme positions of the arms are drawn in blue and red. The dark orange disc shows the direct workspace, which is the locus of the positions where the hand-bot can grasp an object with one gripper fixed. The light yellow zone shows the indirect workspace, which is the locus of all locations where the robot can grasp an object, possibly after climbing up and down (B) Trace of the handbot climbing to fetch a book. The blue lines show the real trajectories of the grippers, extracted from Video 2. The red dotted lines show the trajectories of the grippers, as computed by the robot. 


\section{Experiment: Climb of a shelf and retrieval of a book}

In this experiment, the hand-bot starts lying on the ground with its right gripper attached to a vertical pole, in this case the border of a shelf. The hand-bot first launches the magnet, and then climbs the shelf by switching grippers alternatively, always keeping at least one gripper attached. To do so, the hand-bot rotates its head slowly and coils the rope accordingly using its elevation motor. The hand-bot actively maintains the grippers in parallel with its body, so that it can grasp the border of the shelf or a book. The hand-bot uses the infrared proximity sensors of the gripper and the orientation of the head to decide when to grasp. When the hand-bot reaches a specific altitude $(120 \mathrm{~cm})$, it scans for the book using the proximity sensors of the gripper. When it has grasped the book, it goes down, freely hanging at the rope. Video $1^{1}$ shows this sequence. This experiment lasts for two minutes.

Fig. $8 \mathrm{~B}$ shows the trajectories of the grippers while the hand-bot climbs the shelf and retrieves the book. The figure shows both the real trajectories extracted from Video $2^{2}$ and the trajectories estimated by the robot. The robot computes them using the length of coiled rope, the head orientation, and the arms extensions. Overall, the two trajectories match well. Both the real trace and the estimated one show a vertical displacement when the robot attaches (or detaches) one of its gripper. The reason is that the arm is a parallel structure, so when the robot retracts (or extends) an arm, the projected position of the gripper on the vertical plane moves away from (or moves closer to) the robot.

There are several causes to the differences between the two traces. First, climbing creates high torques on the grippers' joints, which affects the robot's balance that is not perfectly horizontal anymore. In the future, we will solve this problem by fusing in the control the information from an accelerometer. Second, as the robot climbs it displaces itself on the horizontal axis, which creates discrepancies with respect to the assumptions of our cinematic model which considers a vertical rope. However, the passive compliance of the grippers allows the robot to overcome this difference and to climb successfully anyway. In the future, we plan to take this effect into account in our model. Third, we manually marked the centers of the grippers every 0.5 seconds on the video of the experiment, which introduced errors on the positions. In addition, the projection of the three-dimensional scene on the two dimensional sensor of the camera introduces major distortions on the depth axis, and minor distortions on the other axis.

\section{Future work}

The current prototype of the hand-bot is able to autonomously climb a shelf and retrieve a book, provided that the robot knows the approximate position of

\footnotetext{
${ }^{1}$ hand-bot retrieves a book, video 1: http: //www . youtube . com/watch?v=92bLgE6D02g

${ }^{2}$ hand-bot retrieves a book, video 2 : http: //www . youtube. com/watch? v=FFyqf051sik In the video, we move slightly the magnet once it is attached, because the shot of the current prototype is not precise enough. This does not affect the autonomy of climbing.
} 
the book. In the future, we will extend the autonomy of the hand-bot in various directions. First, we will add the main processor board running Linux and the three-megapixel camera to enable the robot to perceive and interact with objects in a more dynamic way. Second, we will explore self-assembling of the hand-bot with ground robots, to provide ground mobility to the hand-bot. This will allow us to implement more complex scenarios and explore deeper scientific questions than what is currently possible with a single hand-bot. Third, we will analyze collaborative tasks involving several hand-bots that manipulate large objects together. Finally, we will perform benchmarks of the collective approach with respect to single-robot solutions, such as humanoids.

\section{Conclusion}

Albeit the use of collective robotics for service applications imposes larger development and introduction costs then an approach based on single robots, it provides specific advantages. These include robustness, flexibility, and scalability that might prove critical for applications such as large deployments of robots in domestic or industrial environments. However, the relationship between the added value in performances and the additional costs in design and development is still unexplored, especially for complex tasks such as the ones involving manipulation. Robots such as the hand-bot provide an engineering foundation to explore this scientific question. The prototype of the hand-bot managed to successfully climb a shelf and retrieve a book autonomously. It achieved this thanks to its innovate synergy between its climbing and manipulation subsystems.

\section{Acknowledgements}

We thank Basilio Noris who shot all the photos of the exterior of the hand-bot and provided us with material and expertise for filming. This work was supported by the Swarmanoid project, which is funded by the Future and Emerging Technologies program (IST-FET) of the European Community under grant IST022888. The information provided is the sole responsibility of the authors and does not reflect the Community's opinion. The Community is not responsible for any use that might be made of data appearing in this publication.

\section{References}

1. IFR Statistical Department: World Robotics 2008. IFR Statistical Department (2008)

2. Konolige, K., Fox, D., Ortiz, C., Agno, A., Eriksen, M., Limketkai, B., Ko, J., Morisset, B., Schulz, D., Stewart, B., et al.: Centibots: Very large scale distributed robotic teams. In: Experimental Robotics: The 9th International Symposium, Springer Tracts in Advanced Robotics (STAR), Springer (2005) 131-140

3. Sahin, E.: Swarm robotics: From sources of inspiration to domains of application. In: Swarm Robotics Workshop: State-of-the-art Survey, Springer (2005) 10-20 
4. Winfield, A.F., Harper1, C.J., Nembrini, J.: Towards dependable swarms and a new discipline of swarm engineering. Swarm Robotics 3342 (2005) 126-142

5. Dorigo, M., Gambardella, L., Mondada, F., Floreano, D., Nolfi, S.: Swarmanoid: Towards humanoid robotic swarms. http://www.swarmanoid.org/

6. Groß, R., Tuci, E., Dorigo, M., Bonani, M., Mondada, F.: Object transport by modular robots that self-assemble. In: Proceedings of the 2006 IEEE International Conference on Robotics and Automation. (2006) 2558-2564

7. Staritz, P., Skaff, S., Urmson, C., Whittaker, W.: Skyworker: a robot for assembly, inspection and maintenance of large scale orbital facilities. In: Robotics and Automation, 2001. Proceedings 2001 ICRA. IEEE International Conference on. Volume 4., IEEE Press (2001) 4180-4185

8. Balaguer, C., Gimenez, A., Huete, A., Sabatini, A., Topping, M., Bolmsjo, G.: The MATS robot: service climbing robot for personal assistance. IEEE Robotics \& Automation Magazine 13(1) (2006) 51-58

9. Hillenbrand, C., Berns, K.: Inspection of surfaces with a manipulator mounted on a climbing robot. In: 37th International Symposium on Robotics (ISR). (2006)

10. Longo, D., Muscato, G.: The alicia3 climbing robot: a three-module robot for automatic wall inspection. Robotics and Automation Magazine, IEEE 13(1) (March 2006) 42-50

11. Luk, B.L., Cooke, D.S., Galt, S., Collie, A.A., Chen, S.: Intelligent legged climbing service robot for remote maintenance applications in hazardous environments. Robotics and Autonomous Systems 53(2) (2005) 142-152

12. Zhang, H., Zhang, J., Zong, G., Wang, W., Liu, R.: Sky cleaner 3: a real pneumatic climbing robot for glass-wall cleaning. Robotics and Automation Magazine, IEEE 13(1) (March 2006) 32-41

13. Aracil, R., Saltaren, R., Reinoso, O.: A climbing parallel robot: a robot to climb along tubular and metallic structures. Robotics and Automation Magazine, IEEE 13(1) (2006) 16-22

14. Vona, M., Detweiler, C., Rus, D.: Shady: Robust Truss Climbing with Mechanical Compliances. In: International Symposium on Experimental Robotics, Springer (2006) 431-440

15. Scheidegger, N., Mondada, F., Bonani, M., Siegwart, R.: Bi-pedal Robot for Rescue Operations. In: 9th International Conference on Climbing and Walking Robots. (2006) 425-430

16. Krishna, M., Bares, J., Mutschler, E.: Tethering system design for Dante II. In: 1997 IEEE International Conference on Robotics and Automation, 1997. Proceedings. Volume 2. (1997)

17. ISO Standard 11898: Road Vehicles Interchange of Digital Information - Controller Area Network - ISO 11898. International Organization for Standardization (1993)

18. Magnenat, S., Retornaz, P., Bonani, M., Longchamp, V., Mondada, F.: Aseba: a modular architecture for event-based control of complex robots (2009) submitted for publication.

19. Magnenat, S., Mondada, F.: Aseba Meets D-Bus: From the Depths of a Low-Level Event-Based Architecture. In: IEEE TC-Soft Workshop on Event-based Systems in Robotics (EBS-RO). (2009) 